\title{
Our Experience of Conscious Sedation as an Anaesthetic Technique in patients undergoing Automatic Implantable Cardioverter Defibrillator
}

\author{
BMM Choudhury, M Rahman,R Sultana, AK Beg \\ Department of Anaesthesiology, NICVD, Dhaka.
}

Key words:

Ventricular

dysrhythmias,

Autamatic

implantable

cardioverter

defibrillator,

Conscious sedation.

\begin{abstract}
:
Ten patients with life threatening ventricular arrhythmias who underwent placement of automatic cardioverter defibrillator (AICD) under conscious sedation are reported. Our clinical experience, patient's satisfaction, recovery profile and complications of the technique of conscious sedation is presented and discussed.

Key words: Ventricular dysrhythmias, Autamatic implantable cardioverter defibrillator, Conscious sedation.
\end{abstract}

(Cardiovasc.j. 2011; 4(1): 42-45)

\section{Introduction:}

An AICD is a small battery-powered electrical impulse generator which is placed in patients who are at risk of sudden cardiac death. It has significantly increased the life expectancy of the patients with life threatening arrhythmias like ventricular tachycardia or ventricular fibrillation. The device is programmed to detect cardiac arrhythmia and correct it by delivering a jolt of electricity. With rapid advancements in the field of interventional cardiology, there has been a constant demand to provide anaesthesia for cardiac patients in the cardiac catheterization laboratory.

Insertion of AICDs is mostly performed in the catheterization suite with only complicated cases referred to the operating room. ${ }^{1}$ "Monitored Anaesthesia Care" is typically chosen with a brief general anaesthesia given for defibrillation testing. General anaesthesia may be chosen for patients with concomitant diseases (e.g.,chronic lung diseases, sleep apnoea) when control of the airway is desired. We used conscious sedation as a technique of choice to provide ideal conditions for the placement of automatic cardioverter defibrillator in ten patients.

Whichever anaesthetic technique is selected, it should be safe, provide ideal conditions during surgical manipulation, defibrillation threshold testing and at the same time there should be quick recovery after the procedure. ${ }^{2}$

Conscious sedation though appears simple, it is one of the most challenging areas in emerging trends in anaesthesiology. It describes a well sedated patient who should remain quiet for two to three hours during the procedure, should respond to verbal comands whenever required and at the same time should not go into deep sleep, respiratory depression or have haemodynamic instability. The objective of this study was to evaluate the safety, efficacy and feasibility of placing AICD in the cardiac catheterization laboratory using conscious sedation.

\section{Methods:}

Patients: Ten patients of either sex (9 males, 1 female) in the age range of 35 to 65 years weighing 50-60 kg were subjected to the study. Eight patients had syncopal ventricular tachycardia as the indication for implanting cardioverter defibrillator. The remaining two had sustained episodes of haemodynamically unstable ventricular tachycardia. Complete cardiology workup including echocardiography, coronary angiography was performed in all patients. Seven patients had the diagnosis of ischaemic dilated cardiomyopathy, two of them had dilated cardiomyopathy of non-

Address of Correspondence: Dr. Begum Marjan Mohol Choudhury, Department of Anaesthesiology, NICVD, Dhaka, Bangladesh. 
ischaemic origin and one had obstructive cardiomyopathy. Most of the patients were receiving ventricular arrhythmia prophylaxis with amiodarone along with other supportive medical treatment. All of the patients received antibiotic prophylaxis of Ceftriaxone $1 \mathrm{gm} \mathrm{I} / \mathrm{v}$ once daily on the day before the procedure.

Pre-anaesthetic check-up was done by the cardiac anaesthesiologist the day before the procedure and assessed for cardiorespiratory status and associated medical diseases. Ejection fraction was in the range of $25-30 \%$ with New York Heart Association (NYHA) class between 2-3. Suitability from the point of view of general anaesthesia were also assessed. They were accepted in American Society of Anaesthesiologists (ASA) grade 3. Informed consent was obtained for the procedure both for conscious sedation and general anaesthesia.

\section{Procedure:}

Following informed consent patients were brought to the cardiac catheterization laboratory after an overnight fast. After establishment of intravenous line, patients were connected to electrocardiogram for continuous monitoring of heart rate and rhythm and arterial oxygen saturation by pulse oximeter. Patch electrodes were fixed in the anterior and posterior positions on the left side of the chest and were connected to automatic external defibrillator. During induction of ventricular fibrillation, it was kept in the manual mode so that in case defibrillation by the device failed, it could be activated and the desired energy could be used to defibrillate the heart.

Under local anaesthesia, right femoral vein was punctured and temporary pacemaker was kept in VVI mode with pacing rate $40-50 / \mathrm{min}$. The conscious sedation was initiated at this stage with an Intravenous injection of midazolam $1 \mathrm{mg}$ and fentanyl citrate $50 \mu \mathrm{gm}$.The time of starting conscious sedation was noted. The degree of sedation was monitored by the Ramsay Sedation Scale $^{3}$. Fentanyl Citrate 25-50 $\mu$ gm was repeated when required to achieve conscious sedation upto level 4 of Ramsay Sedation Scale. 5-6 L/min of oxygen by face mask was administered to maintain arterial oxygen saturation of $99-100 \%$.
Table-I

Ramsay sedation scale ${ }^{3}$

1. Patient is agitated, anxious and restless

2. Patient is cooperative, oriented and tranquil

3. Patient responds to commands only

4. Brisk response to glabellar tap or loud auditory stimulus

5. Sluggish response to glabellar tap or loud auditory stimulus

6. No response to glabellar tap or loud auditory stimulus

Under local anaesthesia (2\% plain lignocane 10-12 $\mathrm{ml}$ ) incision and making subcutaneous pocket and insertion of defibrillation lead through left subclavian vein in the left infraclavicular region was done. The leads were finally positioned in the right ventricular apex under fluoroscopic guidance. After appropriate sensing and pacing the ventricular lead was secured to the pectoral fascia. Five minutes before defibrillation threshold testing another dose of fentanyl citrate $25 \mu \mathrm{gm}$ and propofol in the bolus dose of $0 \cdot 4-0 \cdot 5 \mu \mathrm{gm} / \mathrm{kg}$ was given to keep the patient in a state of deep sedation (level 5 of Ramsay sedation scale). Ventricular fibrillation was induced with high energy stimulus DC shock $12 \mathrm{~J}$ for 2 seconds and automatic defibrillation capability of the device was tested with 15-20 J.Finally the device was activated for detection of ventricular fibrillation and automatic defibrillation based on morphology discrimation before the patient was shifted to the coronary care unit. Patients were monitored continuously for heart rate, blood pressure (non- invasive), arterial oxygen saturation, level of sedation and apnoea. After full recovery from sedation, patients were questioned to assess the degree of amnesia for operative and post-operative events and overall satisfaction with the technique.

\section{Results:}

In our protocol midazolam was used as a basal sedative supplemented with a short acting opioidfentanyl citrate and propofol. The total dose of midazolam was $1 \mathrm{mg}$, fetanyl 100-150 $\mu \mathrm{gm}$ and propofol $0 \cdot 4-0 \cdot 5 \mathrm{mg} / \mathrm{kg}$. The sedation time was $60 \pm 30$ mins. Patients remained relaxed and haemodynamically stable. None of the patients had respiratory depression, apnoea,decrease in oxygen saturation below $96 \%$ and all the patients were cooperative during the procedure. 
All patients were successfully tested for defibrillation except one patient who was defibrillated with external defibrillation through patch electrode with energy of $200 \mathrm{~J}$. No other complications were observed in any of the patient during induction of ventricular fibrillation or during defibrillation threshold testing. No complications such as pneumothorax, ventricular dysrythmias or sudden cardiac death in the immediate post-operative period were seen. No patient complained of pain or awareness during defibrillation except one who was defibrillated by automatic external defibrillator.

\section{Table-II}

Aldrete scoring system

\begin{tabular}{lc}
\hline Activity & Score \\
\hline 1. Ability to move 4 extremities & 2 \\
voluntarily/on command & \\
Ability to move 2 extrimities & 1 \\
voluntarily/on command & \\
Ability to move 0 extrimity & 0 \\
voluntarily/on command & \\
Respiration & \\
Able to deep breathe and cough freely & 2 \\
Dyspnoea or limited breathing & 1 \\
Apnoea & 0 \\
Circulation & \\
BP+ 20\% of pre sedation level & 2 \\
BP+ 20\%-50\% of pre sedation level & 1 \\
BP+50\% of pre sedation level & 0 \\
Consciousness & \\
Fully awake & 2 \\
Arousable to voice & 1 \\
Not responding to voice & 0 \\
Colour & \\
Pink & \\
Pale,dusky,blotchy,jaundiced, other & 1 \\
Cyanotic & 0 \\
\hline
\end{tabular}

\section{Discussion:}

Conscious sedation as described by the American Academy of Paediatrics is a medically induced state of central nervous system depression in which communication is maintained so that patient can respond to verbal commands. ${ }^{4}$ Therefore conscious sedation is the proper administration of drugs to obtund,dull or reduce the intensity of pain and awareness without loss of defensive reflexes such as protection of airways. Conscious sedation is generally achieved when there is slurred speech but the patient is arousable and is able to respond. Three levels of sedation have been described: (a) Light sedation - with the use of oral or intravenous sedatives, hypnotics, anxiolytics light sedation is basically used to reduce anxiety; amnesia may or may not be present. (b) Conscious sedation - is a medically controlled state of depressed consciousness where protective reflexes are intact and the patient's ability to maintain a patent airway and appropriate response to physical stimulation or verbal comand are maintained. (c) Deep sedation - is a medically controlled state of depressed consciousness or unconsciousness from which patient is not easily aroused and is unable to respond purposefully to physical stimulation or verbal comand.

Patient may progress from one degree of sedation to another depending on the underlying medical status, the medications administered, dosage and route of administration. In cardiac patients, the response to sedatives may vary due to altered brain circulation time and pharmacodynamics. Genotype may influence perioperative risk and outcome. ${ }^{5}$ Various drugs that have been used singly or in combination for conscious sedation include: midazolam, fentanil, morphine,propofol,chloral hydrate, droperidol and pethidine. Morphine has been popular but there is always a possibility of deep sedation or respiratory depression. With the availability of short acting benzodiazepines and opioids, technique of conscious sedation has become safer. Propofol can offer an additional advantage due to its short duration of action, fast recovery and anti-emetic properties. Propofol in the bolous dose of $400 \mu \mathrm{g} / \mathrm{kg}$ has been used for defibrillation threshold testing. However it may cause dose dependent fall in blood pressure and apnoea. Propofol infusion in the dose of $20-50 \mu \mathrm{g} / \mathrm{kg} / \mathrm{min}$ can be used safely for maintaining conscious sedation. Thiopentone has also been used for the same purpose but prolonged recovery is observed with thiopentone. ${ }^{6}$ 
With general anaesthesia, there is obviously optimum patient comfort and better airway control but some cardiorespiratory complications in cardiac patients with poor left ventricular functions can not be ruled out. Some of the recent reports have shown 4 percent complication rate directly related to endotracheal intubation and general anaesthesia. ${ }^{7}$ Moreover there may be prolonged hospital stay in those who receive general anaesthesia. 8 The patient's in this series underwent nonthoracotomy placement of automatic ICD under conscious sedation without general anaesthesia. The technique of conscious sedation used was found to be safe and ideally suited for this procedure.

In our protocol of conscious sedation we used midazolam, propofol and fentanyl with no obvious complications except one who required external defibrillation with patch electrode for defibrillation testing (DFT). Pacifio and his associates from Houston, Texas used midazolam $15-30 \mu \mathrm{g} / \mathrm{kg}$ within 20 seconds, promethazine $180-360 \mu \mathrm{g} / \mathrm{kg}$ and morphine $30-90 \mu \mathrm{g} / \mathrm{kg}$ for conscious sedation. To induce deep sedation for defibrillation testing, a bolus of etomidate $80-120 \mu \mathrm{g} / \mathrm{kg}$ within 20 seconds was administered. ${ }^{9}$ VP Singh and his associates from Army Hospital Delhi, India developed a protocol for initiation of conscious sedation with an IV injection of midazolam $1 \mathrm{mg}$ and fentanyl citrate $50 \mu \mathrm{g}$ and when required midazolam 0.5 to $1 \mathrm{mg}$ and fentanyl citrate $25-50 \mu \mathrm{g}$ was repeated as and when required to achieve conscious sedation level up to level 4 of Ramsay sedation scale. Some workers have used another protocol with midazolam 15 to $30 \mu \mathrm{g} / \mathrm{kg}$ followed by propofol infusion in the dose of $20-50 \mu \mathrm{g} / \mathrm{kg} / \mathrm{min}$. During DFT s, propofol bolus in the dose of 400-500 $\mu \mathrm{g} / \mathrm{kg}$ was administered. Tung et al have used midazolam 1-2 mg or fentanyl 25-50 $\mu \mathrm{g}$ or both every 2-5 min depending upon the depth of sedation, which was monitored closely for respiratory pattern, eyelash reflex and spontaneous movements to painful stimuli ${ }^{10}$. The dose of midazolam and fentanyl ranged between 2-4 $\mathrm{mg}$ and 150-300 $\mu \mathrm{g}$ respectively . On follow up, none of their patients recalled pain or shock during the procedure except one, who recalled 1 of 5 shocks during implantation.

We assessed recovery of our patients by applying Ramsay sedation scale. All patients achieved the scale of 2-3 within 15-30 min following conscious sedation. The recovery charecteristics can also be monitored by applying the Aldrete scoring system.
There were no cardiac or respiratory complications due to conscious sedation in any of our patients either during or after the procedure. The brief twitching movements in two patients in whom the defibrillation testing by the device failed, could be due to delay in the charging in the capacitor. With the increasing number of implantations of ICDs, the anaesthesiologists will have greater role to play in the cardiac catheterization / electrophysiological laboratory. The results of our technique of conscious sedation suggest that it is safe, reliable and with no major compromise of cardiorespiratory function.

\section{References:}

1. Michael J. Audritsas, Mitchell Faddis, Charles W.Hogue, Jr. Cardiac Electrophysiology: Diagnosis and Treatment - Implantable Cardioverter-DefibrillatorAnesthetic Consideration. In : Joel A. Kaplan, David L.Reich, Carol L. Lake, Steven N.Konstadt. Eds. Kaplan's Cardiac Anesthesia $5^{\text {th }}$ ed. Philadelphia. Elsevier Saunders, 2006 : 378.

2. Col VP Singh, Lt Gen BN Shahi, Lt Col A Dhali, Brig ML Chawla, Lt Col A Sinha. Conscious sedation as an Anaesthetic Technique in Patients Undergoing Nonthoracotomy Placement of Automatic Implantable Cardioverter Defibrillator: An Initial Experience. Annals of Cardiac Anesthesia 2004; 7: 149-154.

3. Ramay MA, Savege TM, Simpson BR, et al. Controlled sedation with alphaxalone / alphadolone. Br Med J 1974; 2: 656-59.

4. American Academy of Pediatrics. Committee on drugs. Guidelines for the elective use of conscious sedation, deep sedation and general anaesthesia in pediatric patients. Pediatrics 1985; 76: 317-32.

5. Stephan Zeigeler, Byron E, Tsusaki DO, Charles D Collard. Influence of genotype on perioperative risk and outcome. Anesthesiology 2003; 99: 212-219.

6. Emre C, Kamalettin K, Zerrin S, et al. Implantable Cardioverter defibrillator placement in patients with mild to moderate left ventricular dysfunction. Hemodynamic and recovery profile with two different anesthetic techniques used during deep sedation. J Cardiothorac Vasc Anesth 2003; 17 : 613-616.

7. Stricberger SA, Hummel A, Kalbfleisch SJ, et al. Implantation by electrophysiologist of 100 consecutive cardioverter defibrillators with non-thoracotomy lead system. Circulation 1994; 90: 868-872.

8. Pinosky MI, Reeves ST, Fisherman RL, et al. Intravenous sedation for placement of automatic implantable cardioverter defibrillator. J Cardiothorac Vasc Anesth 1996; 10: 764-766.

9. Pacifio A, Felix R, Nasir N, et al. Conscious sedation with combined hypnotic agents for implantation of implantable cardioverter defibrillator. J Am Coll Cardiol 1997; 30: 769-773.

10. Tung RT, Bajaj AK. Safety of implantation of a cardioverter defibrillator without general anaesthesia in an electrophysiology laboratory. Am J Cardiol 1995; 75: $908-912$. 